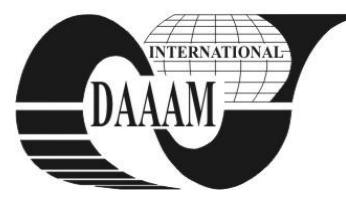

\title{
3D DIGITIZATION OF CURVES ON HUMAN BODIES BY MEANS OF DIGITAL CLOSE RANGE PHOTOGRAMMETRY
}

\author{
PERCOCO, G[ianluca]; GALANTUCCI, L[uigi] M[aria] \& LAVECCHIA, F[ulvio]
}

\begin{abstract}
The realization of custom made clothes implies continuous tests, long times of delivery and often prohibitive costs. In this field, one of the most interesting challenges is to automate some parts of the process to obtain a competitive product.The first step to be completed to automate the realization of customized garment apparels is the $3 D$ Digitization of customers. In the present research the authors present a properly designed low cost offline $3 D$ body digitizer, based on Digital Close Range Photogrammetry. The results are satisfactory for tailoring applications that do not require high accuracy. Furthermore, essays on Dense Surface Models are described
\end{abstract}

Key words: $3 D$ Scanning, textile industry, human digitization, anthropometry

\section{INTRODUCTION}

In the textile industry Reverse Engineering (RE) can be used to achieve the Computer Aided Design (CAD) model of the customer; the CAD model can be sent to the Computer Aided Manufacturing (CAM) interface to automatically generate paths for cutting textiles but also to perform the virtual try-on in order to correct undesired features before realization, reducing the number of tests on the customer. The virtual simulation can avoid physical Prototyping, currently used in the textile industry, with remarkable savings in terms of time and money (Chittaro \& Corvaglia, 2003). The development of 3D CAD model reconstruction of the human body could represent a dramatic improvement towards the mass customization of clothes, accelerating e-commerce through the use of virtual mannequins. Several examples are findable in literature regarding advanced methods of manufacturing clothes from 3D scanned body data (Kim \& Park, 2007; Percoco, 2011; Kim \& Kang, 2002). The development of 3D Scanning technologies is being exploited by modern anthropometry revealing the shape and the changes due to time in the human body, allowing genetic or acquired malformations to be diagnosed in a less invasive way, surgical treatments be planned more deeply, normal and abnormal growth be observed verify the results of therapies. One example is given by orthodontics, where analyses of patient uses radiographic images and conventional photos to estimate three-dimensionally the face, and to formulate clinical diagnosis. Standard photos are not enough for diagnosis and often fail clinical requirements. A notinvasive method, that uses only clinical photographs, still does not satisfy clinical requirements.

\subsection{BODY SCANNERS}

3D digitization of human bodies is used in several sectors of knowledge and can be subdivided into two main groups: a) the first group is mainly related to the visual results: cinematographic industry, computer games, art and virtual reality; b) the second group aims to the quantitative aspect of the results. As regards textile applications, the most used digitizing systems can be classified basing on the technologies used such as Laser Scanning, structured light projection, Image elaboration and modeling, microwaves. For an exhaustive analysis of several $3 \mathrm{~d}$ scanning technologies, please refer to (Kang \& Kim, 2000).

Systems based on laser triangulation allow to measure three-dimensional surfaces by means of a simple apparatus constituted of laser emitter and two CCD cameras. The number of laser sources depends on the dimensions of the part of the body to be digitized. Several scanners are actually on the market for the acquisition of the entire human body, based on this technique. They differ mainly in the way of projection of the laser beam and in the way the image is captured. The majority of scanners (Cyberware, Vitronic, TecMath, Hamamatsu) projects on the body horizontal strips and takes advantage of the vertical movement of the laser head. Other typologies (Hamano Engineering) work with two rotary mirrors projecting vertical strips over the body.

As regards scanning time and quality, it is very important to block the head of the subject; in this case the scanning errors are reduced of $50 \%$ approximately, while the scanning time is reduced of about $30 \%$. The scanning time of commercial laser scanners can vary between 5 and 20 seconds. Medical scanners have much better performances than those requested by the apparel industry, achieving an accuracy approximately equal to 0.2 millimeters and acquisition time approximately equal to 0.3 seconds. The costs of these technologies are still very high and this is reflected in the selling price of the body scanners, that can reach several hundreds of thousands Euros. The high price of this type of scanner is a strong disadvantage, as it limits very much the diffusion of this technology in the textile field.

Instantaneous 3-D imaging systems, based on photogrammetry, where complete 3-D information are gained with the acquisition and matching of several images. These methods are particularly suited for the digitization of human body data because of their insensitivity to slight body movements. In order to create a model of the body, the 3-D information can be directly acquired or a generic pre-existing model of the face may be employed, then adapted to the specific person.

According to the authors, the photogrammetric technique introduce the best margins of improvement, owning intrinsic characteristics such as very low scanning times, low cost equipment and no intrusiveness.

\section{THE PHOTOGRAMMETRIC 3D SCANNER}

In this paper it is proposed one specifically designed scanning system composed of 8 usb video cameras with resolution equal to 5 Mpixels, equipped with $16 \mathrm{~mm}$ wide lenses; 4 white light illuminators, $100 \mathrm{~W}$ each. The elaboration of the images has been carried out through the software Photomodeler 6.0 by Eos Systems. The calibration of the system has been performed using one $12 \times 12$ points grid with 4 coded points. Six images per camera have been used, for a total of 48 photos. 


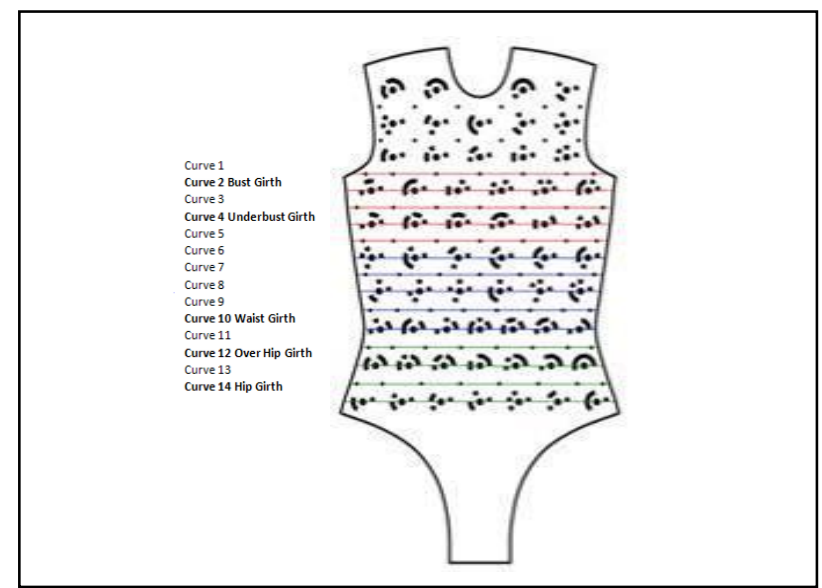

Fig. 1. The photogrammetric corset

Coded Targets are points with additional bits that can be automatically recognized by the software during the marking process. This automatic recognition allows for automated referencing even before a project has been processed and in complex scene or shape. Coded Targets increase the speed at which projects can be completed, improve the speed and robustness of the crucial orientation stage which all projects must go through at the start, and improve robustness and reduce the chances of misreferenced points. The dimensions of the coded targets are very important to have a correct referencing and digitization.

\subsection{Single point photogrammetry}

The scanner has been preliminarily validated through several tests, namely tests on dummy busts, with an Italian size 42 (correspondent to an American size 8). The busts have been dressed with several specially designed corsets leading to the choice of the hybrid coded-non coded targets corset shown in Fig. 1. The digitized points have been joined together using spline curves computed by a commercial CAD software and the length of each curve has been pointed out. This process has been performed both on a dummy and a human subject.

The results related to the dummy have led to an average error on the measures equal to $0,49 \%$ measured on 225 digitized points. As regards the human subject the results are worse than those on the dummy, with a mean error equal to $0.56 \%$, computed on 193 points.

\subsection{Dense Surface Modelling}

In order to compare the accuracy of the photogrammetric method to laser scanning, Dense Surface Modeling (DSM) have been used. A Dense Surface Model is a digital representation of a shape and is used in any application where a large number of 3D points (a point cloud) is needed. The DSM process creates a

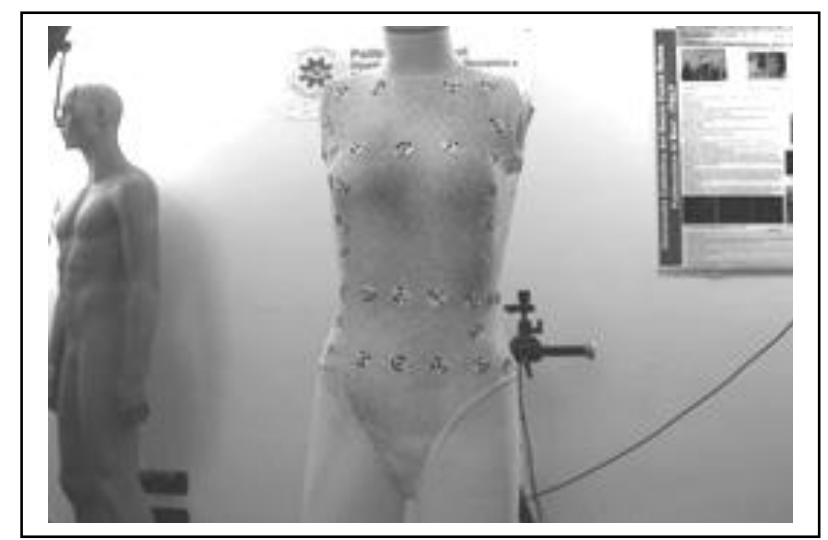

Fig. 2. The corset designed for DSM

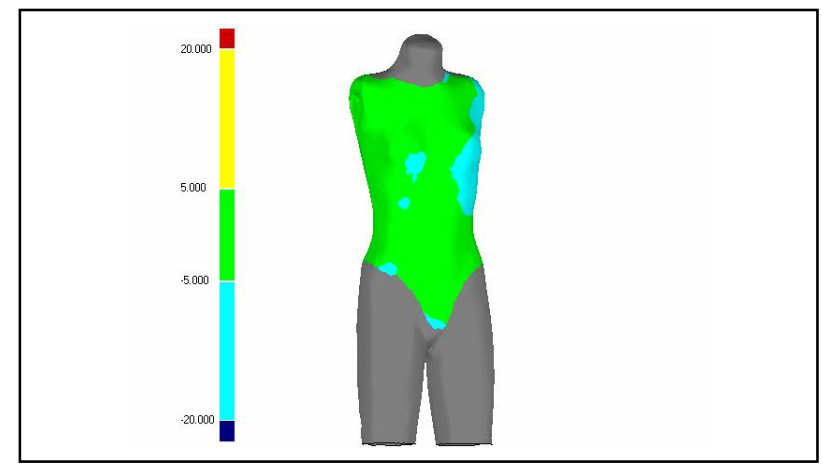

Fig. 3. 3D Comparison laser-DSM

dense set of points with optional triangles connecting them.

When a good match is found between two photos, the orientation and camera data allows the program to compute the Dense Surface Modeling is a search algorithm that uses an existing pre-oriented project and pairs of photos from that project to search for image patches that 'look' alike. This search is done in a regular grid-like manner so a grid of 3D points is computed. To achieve the results the object must be textured and, as a consequence, a different corset has been designed, using coded targets in critical areas of a grey textured corset (Figure 2). The first pair of cameras led to 109,544 points, the second 51,828 , the third 117,328 and the fourth 47,578 , for a total of more than 300,000 points. The average values of the deviations are equal to 1.50 and $-4.50 \mathrm{~mm}$. In Figure 3 it is shown a colored map that compares the measure performed with DSM and the laser scanner Minolta Vivid 910i. It is possible to verify comparable results of accuracy.

\section{CONCLUSION}

The experimental 3D scanner presented in this paper confirms the usefulness of photogrammetry with coded targets for digitalization of human bodies. The three-dimensional information regarding the dummy bust are correctly retrieved. In order to better evaluate the accuracy of the process, a preliminary comparative test with laser scanning has been described with satisfactory results. The main limitation of the present research consists in the use of properly designed corsets and further research will be carried on to avoid the need of dressing these corsets to retrieve a good quality point cloud.

\section{REFERENCES}

Chittaro, L., Corvaglia, D. (2003). 3D Virtual Clothing: from Garment Design to Web3D Visualization and Simulation, Proceedings of Web3D 2003, 8th International Conference on 3D Web Technology, April 2003, ACM Press, New York, pp. 73-84, available at http://hcilab.uniud.it/publications/2003-04/3DVirtualClothing_WEB3D03.pdf, last accessed on 27/9/2011

Kang T. J, Kim S. M..(2000). Optimized garment pattern generation based on three-dimensional anthropometric measurement, International Journal of Clothing Science and Technology, Vol. 12 Issue 4, Sept. 2000, pp. 240-254, ISSN: 0955-6222

Kim, S., Kang, T. (2003). Garment pattern generation from body scan data, Computer-Aided Design, Vol. 35, Issue 7, June 2003, pp.611-8. ISSN 0010-4485

Kim, S.; Kyu P. C..(2007). Basic garment pattern generation using geometric modeling method, International Journal of Clothing Science and Technology, Vol. 19 No. 1, Jan 2007, pp. 7-17, ISSN: 0955-6222

Percoco G. (2011). Digital close range photogrammetry for 3D body scanning for custom-made garments, The Photogrammetric Record, Volume 26, Issue 133, March 2011, pp 73-90, ISSN: 0031-868X 